\title{
Selective Effects of Fatty Acids Upon Cell Growth and Metabolic Regulation
}

WILLIAM E. M. LANDS, ROGER W. SACKS, and JOHN SAUTER, Department of Biological Chemistry, The University of Michigan, Ann Arbor, Michigan 48109 and FRANK GUNSTONE, Department of Chemistry, University of St. Andrews, St. Andrews, Scotland

\begin{abstract}
Positional isomers of cis-methyleneoctadecanoic acid differed greatly in their efficiency for growth of an unsaturated fatty acid auxotroph of Escherichia coli upon glucose as a carbon source. The 8, 9, and 11 isomers were more efficient in producing cells $(60-70$ cells/fmole) than the others $(0-7$ cells/ fmole), although all isomers were found esterified to a similar extent into cellular lipid. With Saccharomyces cerevisiae mutants, all isomers between 6 and 12 supported some grow th of the eukaryotic cells, and the 7 and 9 isomers were slightly more efficient than the 8 -isomer. When $E$. coli were grown with glycerol, all isomers from 5 to 14 supported growth, and those with the substituent near the center of the acyl chain had the greatest efficiency ( 70 cells/fmole). With the glycerol medium, the pattern of efficiencies for the various cis-methylene acyl chains resembled the broad selectivity reported earlier for the cis-ethylenic isomers in glucose medium, which agreed closely with predictions based upon the physical property of their phospholipid derivatives. Thus, metabolism of glycerol appeared to allow the cyclopropane acyl chains to support cell functions to the limits expected for bulk phase chain-chain fluidity considerations. This broad specificity was also obtained when cells were grown on glucose with cyclic AMP added to the culture. Therefore, the selective inadequacies of the $5,6,7,10,12$ and 13 isomers in supporting cell growth on glucose may occur through an interaction modified by cAMP and dependent upon reduced cellular levels of cyclic AMP. The highly selective pattern of efficiency of the cis-methylene acids for $E$. coli growth on glucose resembles that with the acetylenic acids, but was shifted one carbon atom toward the methyl terminus. This observed selectivity pattern seems due to interactions of the individual acyl chains with cellular protein(s) rather than to chain-chain interactions in a bulk phase. The ability of certain positional isomers to support cell function equally well in both nutrient conditions suggests that the role of those acyl chain isomers may be independent of metabolite flux or cyclic nucleotide contents of the cell, whereas the actions of other isomeric fatty acids seem closely related to the metabolic status of the cell. A highly selective role for different fatty acids in modulating cellular function seems possible on the basis of the current evidence.
\end{abstract}

\section{INTRODUCTION}

The acyl chains of cellular lipids are commonly assigned a significant role in maintaining the integrity of the membrane bilayers (1). Combinations of saturated and unsaturated acyl chains presumably can provide favorable degrees of expansion, plasticity or fluidity that facilitate membrane-mediated processes. An essential role for unsaturated fatty acids in cell physiology is evident in studies with auxotrophic microbial mutants that cannot synthesize the unsaturated bond $(2,3)$ and thereby cannot grow without supplemental nutrient unsaturated fatty acids. Such organisms provide useful model systems for determining the manner by which acyl chains play their vital roles.

A wide variety of acyl chains occur in nature, and the more complex eukaryotic organisms have evolved a series of dehydrogenases which introduce cis-ethylenic bonds into preformed acyl chains to give many different acids in the cellular lipids (4). The eukaryotic acylCoA:phospholipid acyltransferases have a selectivity (5) that ensures rapid incorporation (and thereby persistent retention) of long-chain polyunsaturated acids in tissue phospholipids. An important role in mammalian cells for some of these acids may be as a precursor of the very potent autacoids, prostaglandins and thromboxanes (6). The need for such autacoids to mediate and modulate complex, integrated eukaryotic cell functions may explain the recognized need by mammals (7) for the $(n-6)$ class of "essential" fatty acids. For example, esterified linoleate [18:2(n-6)] could be regarded as an esterified pool of precursor for forming eicosatrienoate $[20: 3(n-6)]$ and arachidonate [20:4(n-6)] which can in turn form PGE $_{1}$ and $\mathrm{PGE}_{2}$, respecitively. However, many additional fatty acids occur for which a distinct physiologic role has yet to be assigned, and the consequence(s) of their occurrence in a cell are uncertain. In addition, we still do not know the degree to which arachidonate (or other (n-6) acids) are aiding cells by forming prostaglandins in comparison to contributing to membrane fluidity. Apparently, an octadecenoate with only one cis-ethylenic bond can provide ade- 
quate plasticity or fluidity for growth of the microbial mutants studied $(8,9,10)$. The value of having the variety of elongated and desaturated acyl chains that are commonly seen in the cellular lipids of eukaryotic organisms is not clearly evident.

We have developed evidence to show that an unsaturated fatty acid may influence cellular functions in a manner other than that expected from contributing to membrane fluidity or from forming prostaglandins and related autacoids. Our previous studies (11-13) indicated that some fatty acids exhibit effects that do not fit the additive, nonselective properties attributable to chain-chain interactions that are associated with fluidity. The current report illustrates that cyclopropane fatty acids can interact with cells in a highly selective manner that affects cell proliferation and that depends upon other aspects of cellular metabolism.

\section{MATERIALS AND METHODS}

The Escherichia coli mutant $30 \mathrm{E} \beta \mathrm{x}^{-}$(14) was obtained from Drs. C.D. Linden and C.F. Fox (Dept. of Bacteriology, UCLA). It was derived from a $\beta$-glucoside-fermenting derivative of $\mathrm{K}-12$ strain MO, $\mathrm{F}^{-} \operatorname{str}^{\mathrm{r}}$ (15) by nitrosoguanidine mutagenesis and penicillin selection to obtain strain $30^{-}$, an unsaturated fatty acid auxotroph lacking the $\beta$-ketoacyl acyl carrier protein synthetase (fab $B$ ), but not lacking the $\beta$-hydroxydecanoyl thioester dehydrase (fab A) $(16,17)$. Subsequently, an elaidate-utilizing mutant $(30 \mathrm{E})$ was derived from $30^{-}(18)$ as described by Schairer and Overath (19), and mutagenized by nitrosoguanidine to give, following penicillin selection, the $30 \mathrm{E} \beta \mathrm{Ox}^{-}$strain (14) used in these studies. The cells showed a 100 -fold reduction in release of ${ }^{14} \mathrm{CO}_{2}$ from carboxy labelled fatty acid when compared to the parent $30 \mathrm{E}$, and showed no $\beta$-oxidation products derived from exogenous fatty acids upon gas chromatographic analysis of the cellular phospholipids (14). The unsaturated fatty acid requirement was routinely validated by the lack of growth on replicate plates without oleic acid, and the absence of functional $\beta$-oxidation was shown by a lack of growth on plates containing oleic acid as the only carbon source.

The medium used, as in the previous studies $(11,12)$, was Medium A supplemented with $0.5 \%$ casamino acids (Difco, Detroit, MI) and either $1 \%$ glucose or $0.5 \%$ glycerol as the energy source. Nutrient unsaturated fatty acids were synthesized as described in an earlier publication (20), and added as the ammonium soaps in ethanol. The solid medium used was medium A with $0.4 \%$ sodium succinate plus $0.1 \%$ glucose as the energy source, $1.5 \%$ agar, $0.5 \%$ Tween- 40 (Sigma, St. Louis, MO) and when desired, $0.15 \%$ oleic acid. The lack of casamino acids in the solid medium also provided conditions to prevent appearance of spontaneous amino acid auxotrophs.

All growth studies were performed at $37 \mathrm{C}$. Culture tubes were inoculated at $10^{7}$ cells per $\mathrm{ml}$ with $\log$ phase cells, and growth was monitored turbidimetrically at $660 \mathrm{~nm}$ with a Bausch \& Lomb Spectronic 20. Microscopic counts were made concurrently with A660 readings during growth and at the harvest of the culture. From these data, a computer-fitted polynomial regression showed cells $/ \mathrm{ml}=-.004+$ $4.47 \mathrm{~A}_{660}+34.56 \mathrm{~A}_{660}^{2}-67.05 \mathrm{~A}_{660}^{3}+42.46$ $\mathrm{A}_{660}^{4}$. Periodic checks of this relationship confirmed the validity of the more convenient turbidimetric readings. In addition to monitoring cell number, aliquots of the cultures were plated on control plates described above at various times during the growth and at stationary phase to check for the presence of possible contaminants or revertants. No contaminants or revertants were observed.

Yeast mutants, Saccharomyces cerevisiae KD-46 and KD-115, were obtained from Dr. A. Keith (Department of Biophysics, Pennsylvania State University). KD-46 is a respiratory deficient mutant (21) that cannot synthesize the porphyrin (22) needed for unsaturated fatty acid synthesis, and KD-115 has respiratory activity, but is deficient in the desaturase (23). The KD-115-PR respiratory-deficient strain is a petite mutant isolated from the KD-115 (Graff and Lands, unpublished). The yeast cultures were maintained with the media and conditions described previously $(24,25)$.

\section{RESULTS}

Increasing amounts of the cylopropane fatty acids gave increased cell yields of both types of cells used. With $E$. coli, only the 8,9 , and 11 isomers produced net cell yields greater than $10^{9}$ cells/ml (Fig. 1). The effectiveness $(\epsilon)$ of each acyl chain in supporting bacterial cell growth was determined from the slope of the cell yield response curves between 10 and 30 $\mu \mathrm{M}$ nutrient, and averaged values for the different positional isomers are compared in Figure 2. The results indicate that isomers 2 to 7 and 12-17 were very ineffective in supporting growth on glucose. Also, the 10-isomer differed greatly from the adjacent isomers (9 and 11). All isomers were found esterified in cellular phospholipids (Table I), and the amount at stationary phase was similar for most isomers whether expressed as mole percent of total 


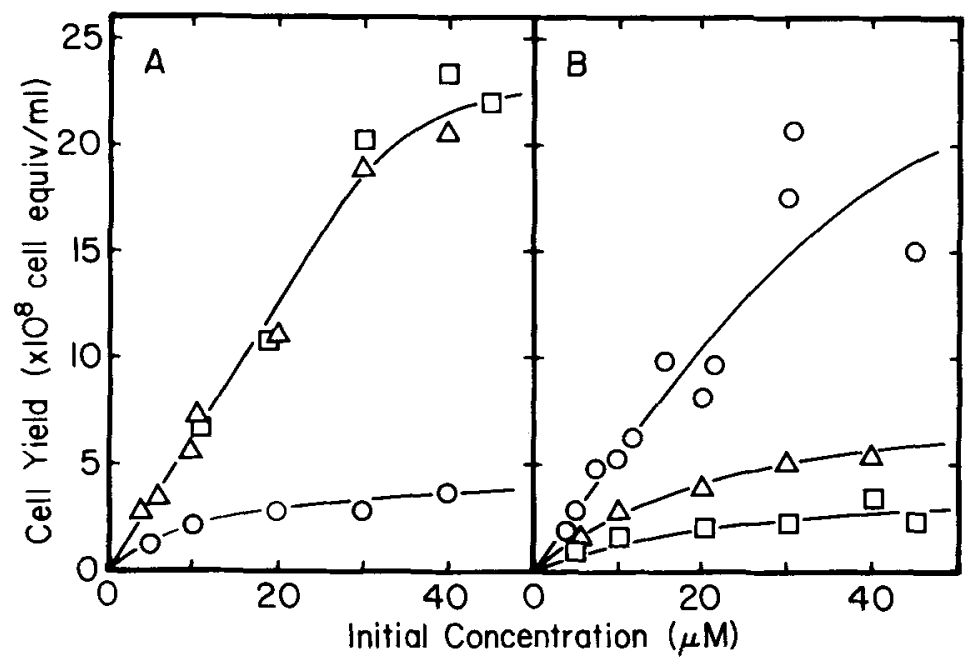

FIG. 1. Cyclopropane acids support growth on glucose. The effect of increasing concentration of cismethyleneoctadecanoic acid isomers on the net cell yields of $E$. coli $30 \mathrm{E} \beta \mathrm{Xx}^{-}$was measured at $37 \mathrm{C}$ with $1 \%$ glucose as the energy source. Values for the odd-numbered isomers (panel A) are noted: 7,$0 ; 9,0 ;$ and $11, \Delta$. The even-numbered isomers (panel B) are noted: 8,$0 ; 10, \square ;$ and $12, \Delta$. Each data point represents the average of several determinations at any one concentration of nutrient acid. Inocula were prepared as previously described (12).

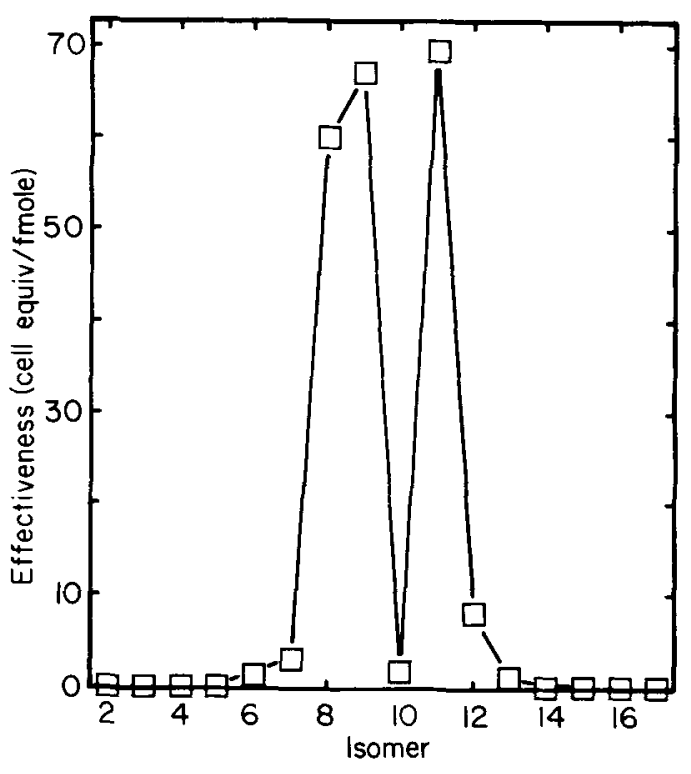

FIG. 2. Effectiveness of cis-methyleneoctadecanoic acid isomers in supporting grow th. E. coli $30 \mathrm{E} \beta \mathrm{x}^{-}$was grown at $37 \mathrm{C}$ with $1 \%$ glucose as the energy source. The ordinate value for each nutrient isomer represents the magnitude of the slope of the linear region of plots of net cell yield vs. initial fatty acid concentration such as those shown in Figure 1.

esters or as amoles of nutrient acid esterified per cell. The values of these contents were greater for the isomers with the substituent near the methyl end of the acyl chain.

Mutants of the yeast $S$. cerevisiae were also able to use cyclopropropane fatty acids to accommodate a nutritional requirement for unsaturated fatty acids. From graphs similar to Fig. 1 (results not shown), the linear regions of increase in cell yield with increased nutrient concentration gave efficiency values $(\epsilon)$ shown in Fig. 3. With all three yeast mutants tested, isomers with the substituent near the center of the chain were the more effective ones. The pattern of selectivity with the respiratory incompetent mutant, KD-46, resembled that with the respiratory competent, KD-115, although the latter mutant formed about 3-fold more cells per fmole of nutrient acid and also grew more effectively with the 12 to 15 isomers. One unexpected difference between the KD-115 (Fig. 3A) and its derived petite mutant (Fig. 3B) was the greater number of cells per fmole produced by the parent strain (KD-115) with the 8 to 10 isomers.

Growth of the bacteria on glycerol provided linearly increased cell yields over a wide range of nutrient fatty acid concentration as shown by the examples in Fig. 4A. The growth effectiveness $(\epsilon)$ of the 8,9 and 11 isomers was equal to that seen for growth on glucose, whereas isomers $5,6,7,10,12,13$ and 14 supported much more growth on glycerol than on glucose (Fig. 4B). The pattern of the $\epsilon$ values for the various isomers was a fairly smooth curve with an optimum for the 10- 
isomer and with none of the sharp irregularities observed for growth on glucose (dashed line).

To test for the involvement of cAMP in the growth response with different carbon sources, cells were grown on glucose plus $1 \mathrm{mM}$ cAMP. Fatty acid isomers observed to be ineffective for growth on glucose alone supported linear cell yields with 10-fold higher efficiencies in the presence of added CAMP (Fig. 5A). The pattern of $\epsilon$ values in the presence of the cyclic nucleotide (Fig. 5B) differed greatly from the pattern on glucose alone, but was almost identical to that shown in Fig. 4B for growth on glycerol. The pattern for growth efficiency on glucose with added cAMP also closely resembled the "bell-shaped" curve reported earlier (12) for growth with the various cis-octadecenoate isomers on glucose with one exception being the inability of the 6-cis-octadecenoate isomer to support growth on glucose. We found that when cAMP was added to the culture medium, the effectiveness of the 6-isomer became nearly 10-fold greater (Fig. 6), whereas that for many other cis-octadecenoate isomers was unchanged.

Analysis of the lipids from cells grown to stationary phase with cyclopropane acids and glucose plus added cAMP produced results (Table II) very similar to those obtained in the absence of added nucleotide. The amoles of total esterified acid per cell ranged from 101 to 190 (compared to 134-198 in unsupplemented), and the amoles of nutrient acid per cell was 7 to 36 (compared to $5-33$ in unsupplemented). In both experimental conditions, the positional isomers with the methylene bridge near the center of the chain were present at lower amounts per cell than were those isomers with the substituent nearer the ends of the chain.

\section{DISCUSSION}

The possibility that fatty acids may exert selective effects beyond their accepted nonselective role in fluidity led us to develop quantitative estimates of the contribution (functionality factor; ref. 25) of the esterified acyl chains to cellular function. We reported a close quantitative agreement between the functionality factor calculated for isomeric cisoctadecenoic acyl chains esterified in cellular membranes and the degree of expansion (or fluidity) of their synthetic phospholipid derivatives (12). The functionality factors calculated for the various cis-ethylenic isomers formed a rather smooth curve with an optimum for the 9- and 10-isomers (12). The nutritional effectiveness $(\epsilon)$ of the different $c i s$-octadecenoates gave a pattern similar to that for the function-
TABLE I

Incorporation of the cis-Methyleneoctadecanoic Acid Isomers into Cellular Lipids of $E$. coli $30 \mathrm{E} \beta \mathrm{xx}^{-}$When Grown on Glucose ${ }^{\mathrm{a}}$

\begin{tabular}{|c|c|c|c|c|}
\hline \multirow[b]{2}{*}{ Isomer } & \multicolumn{4}{|c|}{ Acids in Cellular Phospholipids } \\
\hline & $\begin{array}{r}\text { To } \\
\text { amole }\end{array}$ & $\begin{array}{l}\text { tal } \\
\text { s/cell }\end{array}$ & $\begin{array}{c}\text { Mole \% } \\
\text { nutrient }\end{array}$ & $\begin{array}{c}\text { Nutrient } \\
\text { amoles/cell }\end{array}$ \\
\hline 4 & 140 & (2) & 3.5 & 5.0 \\
\hline 5 & 134 & (2) & 7.0 & 9.4 \\
\hline 6 & 150 & (2) & 6.7 & 10.0 \\
\hline 7 & 175 & (4) & 8.0 & 14.0 \\
\hline 8 & 163 & (4) & 8.7 & 14.3 \\
\hline 9 & 158 & (4) & 8.0 & 12.7 \\
\hline 10 & 175 & (4) & 7.3 & 12.7 \\
\hline 11 & 182 & (4) & 8.2 & 14.9 \\
\hline 12 & 198 & (4) & 11.4 & 22.7 \\
\hline 13 & 164 & (2) & 15.9 & 26.1 \\
\hline 14 & 148 & (2) & 22.0 & 32.6 \\
\hline 15 & 158 & (2) & 11.4 & 18.0 \\
\hline
\end{tabular}

aThe content of nutrient acid in the total phospholipid was determined by GLC on $10 \%$ diethyleneglycol succinate at $180 \mathrm{C}$ utilizing a Varian 2740 Gas Chromotograph. Cultures were supplemented with concentrations $(10-20 \mu M)$ of the indicated isomer and harvested and extracted as described earlier (12). Values in parentheses indicate the number of determinations made.

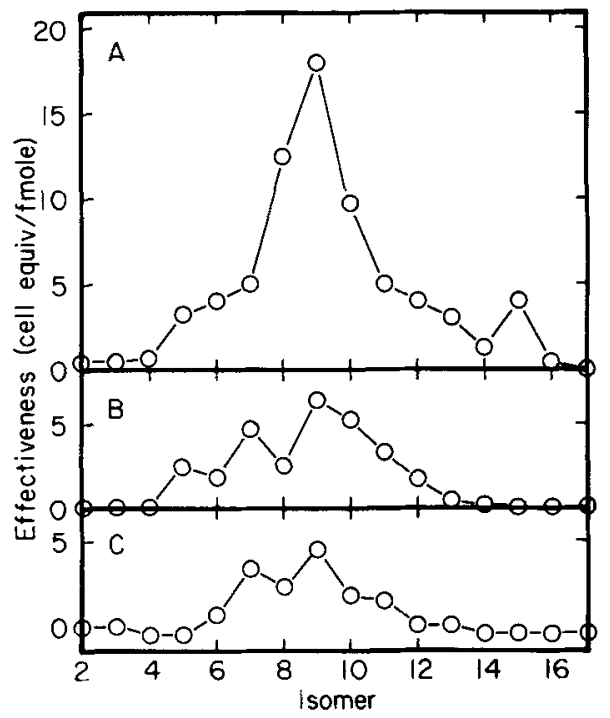

FIG. 3. Effectivenss of cis-methyleneoctadecanoic acid isomers in supporting growth of Saccharomyces cerevisiae. The ordinate value for the efficiency of each isomer was determined as described in Figure 2. Preparation of inocula and growth conditions have been described elsewhere (19). For each mutant, the energy source was glucose. KD-115 (panel A), KD-115 PR (panel B), and KD-46 (panel C).

ality factors with one exception: the 6-isomer. Thus, the cis-6-octadecenoate seemed to be involved in some selective interaction that was 

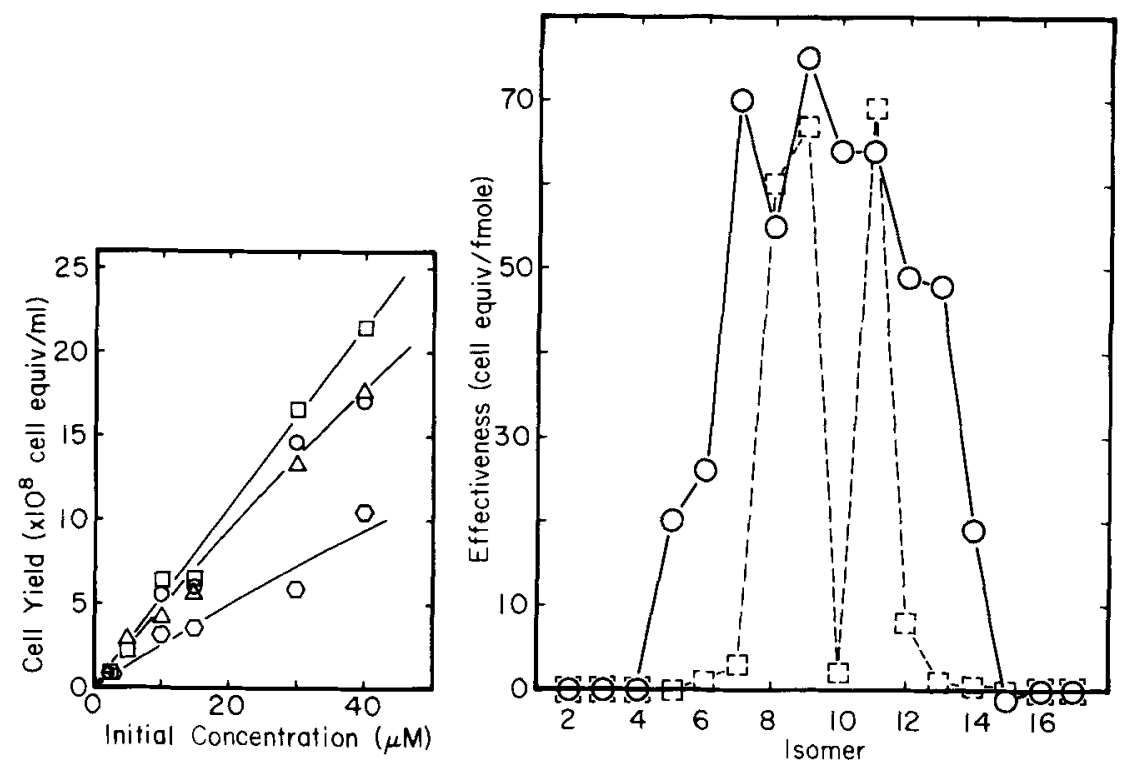

FIG. 4. Cyclopropane acids support growth on glycerol. A. The effect of increased initial fatty acid concentration of the even-numbered isomers of cis-methyleneoctadecanoic acid on the net cell yield with $0.5 \%$ glycerol as the energy source for the $E$. coli mutant at $37 \mathrm{C}$. Data were obtained with inocula and growth conditions (except carbon sources) as described in Figure 1 . Symbols: $6, \bigcirc ; 8,0 ; 10, \square ;$ and $12, \triangle . B$. Comparison of the efficiencies of the cis-methyleneoctadecanoic acid isomers for supporting growth of the $\dot{E}$. coli mutant with either $0.5 \%$ glycerol $(\mathrm{O}-\mathrm{O})$ or $1.0 \%$ glucose $(\square-\square)$ as the energy source.
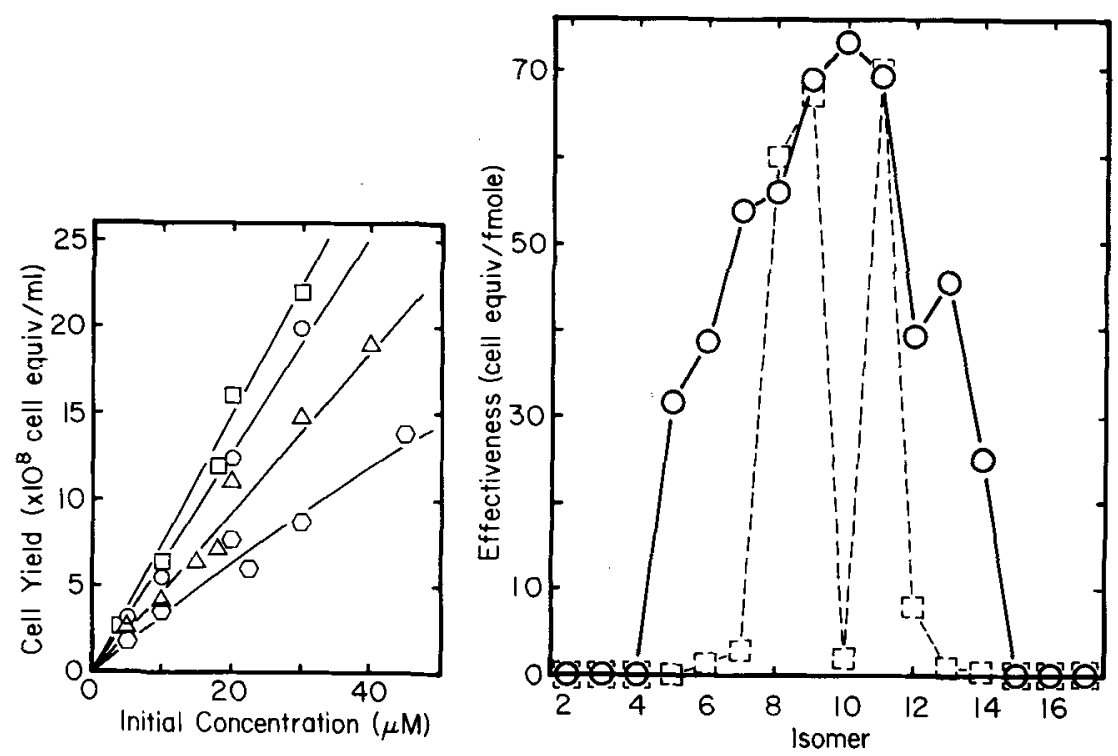

FIG. 5. Modification of acyl chain effectiveness by added cAMP. A. The effect of added cAMP (1 $\mathrm{mM})$ on the net cell yields of the $E$. coli mutant at $37 \mathrm{C}$ with selected even-numbered $c i s$-methyleneoctadecanoic acid isomers and $1 \%$ glucose as the energy source. Symbols: $6 . \bigcirc ; 8,0 ; 10,0 ;$ and $12, \triangle$. B. The efficiencies of the cis-methyleneoctadecanoic acid isomers to support growth of the E. coli mutant when cAMP (1 mM) was added to cultures with $1 \%$ glucose as the energy source $(\mathrm{O}-\mathrm{O})$ is compared to the corresponding efficiencies obtained for growth with glucose alone ( $\square-\square)$. 


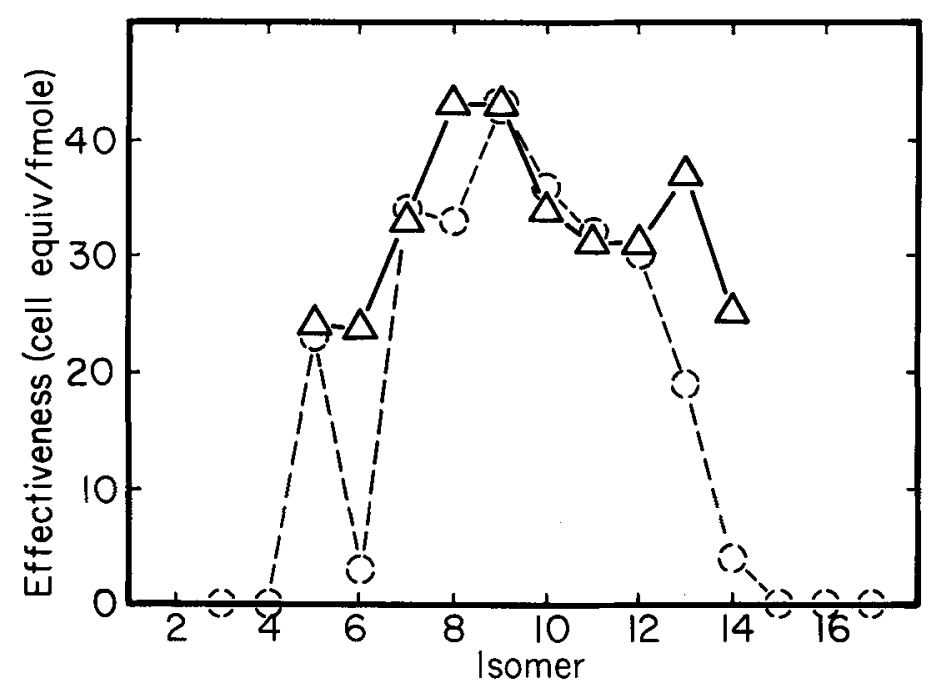

FIG. 6. Modification of selective growth responses by added cAMP. Comparison of the effectiveness of selected cis-octadecenoic acid isomers for supporting growth of the $E$. coli mutant at $37 \mathrm{C}$ with $1 \%$ glucose when supplemented with $1 \mathrm{mM}$ cAMP, $\Delta-\Delta$; and glucose alone, $0-0$.

restraining cell growth in a way not expressed by the other cis-octadecenoate isomers. Selective effects are manifested in two ways: (a) a lower than normal ratio of the nutrient effectiveness $(\epsilon)$ to the calculated functionality factor (f), and (b.) a low maximum cell yield that becomes independent of increased nutrient acid concentration while still below that obtained with normal fatty acids (as shown in Fig. 1). Our studies were designed to quantitatively compare acids under conditions where the cells have just ceased growth as a result of insufficient nutrient fatty acid. Under these conditions, an acid that is highly effective in supporting growth will produce many cells per fmole and therefore be present at a small number of amoles per cell (25). Thus, a low concentration of nutrient acid at stationary phase may not indicate that the acid is an ineffective nutrient, but that it is actually a very effective one that can support further cell division when present at a concentration below that where other fatty acids may fail. The higher content (mole \% and amoles nutrient per cell) observed for isomers with the functional group near either end of the acyl chain (particularly the methyl end) is consistent with the recognized lower contribution to fluidity in bulk phase of the corresponding cis-ethylenic analogs (12,26), and reaffirms the presumed importance of fluidity in supporting cell replication. Those isomers (at positions, 6 , 7,10 , and 12 ) with low ratios of $\epsilon / f$ provide evidence for selective growth-limiting events that are due to processes other than fluidity.
TABLE II

Incorporation of cis-Methyleneoctadecanoic Acid Isomers Into Cellular Phospholipids of $E$. colt 30 EBox when Growth on Glucose in the Presence of Added CAMP3

\begin{tabular}{ccccc}
\hline & \multicolumn{3}{c}{ Acids in Cellular Phospholipids } \\
\cline { 2 - 4 } Isomer & $\begin{array}{c}\text { Total } \\
\text { amoles/cell }\end{array}$ & $\begin{array}{c}\text { Mole \% } \\
\text { nutrient }\end{array}$ & $\begin{array}{c}\text { Nutrient } \\
\text { amoles/cell }\end{array}$ \\
\hline 4 & 112 & $(2)$ & 6.1 & 6.8 \\
5 & 172 & $(2)$ & 15.6 & 26.8 \\
6 & 185 & $(2)$ & 13.7 & 25.3 \\
7 & 154 & $(2)$ & 7.0 & 10.8 \\
8 & 161 & $(2)$ & 8.5 & 13.8 \\
9 & 153 & $(1)$ & 8.0 & 12.2 \\
10 & 171 & $(2)$ & 8.5 & 14.6 \\
11 & 101 & $(1)$ & 4.4 & 4.4 \\
12 & 190 & $(2)$ & 6.1 & 11.7 \\
13 & 160 & $(2)$ & 7.1 & 11.4 \\
14 & 154 & $(2)$ & 30.1 & 46.4 \\
15 & 128 & $(2)$ & 23.0 & 29.5 \\
\hline
\end{tabular}

${ }^{a}$ Conditions and procedures were the same as in Table I with $1 \mathrm{~m} M$ cAMP added to the medium.

\section{Conformational Aspects}

Many acetylenic analogs exhibted a highly selective inability to support bacterial growth (11) that was not predicted on the basis of general physical-chemical considerations (which tend to be nonselective and additive). Figure 7 illustrates the striking similarity of the growth efficiencies for the acetylenic and cyclopropane fatty acids. The patterns for the two types of fatty acid isomers are almost superimposable with the exception of a shift of one carbon atom. We first described the "frame-shift" 


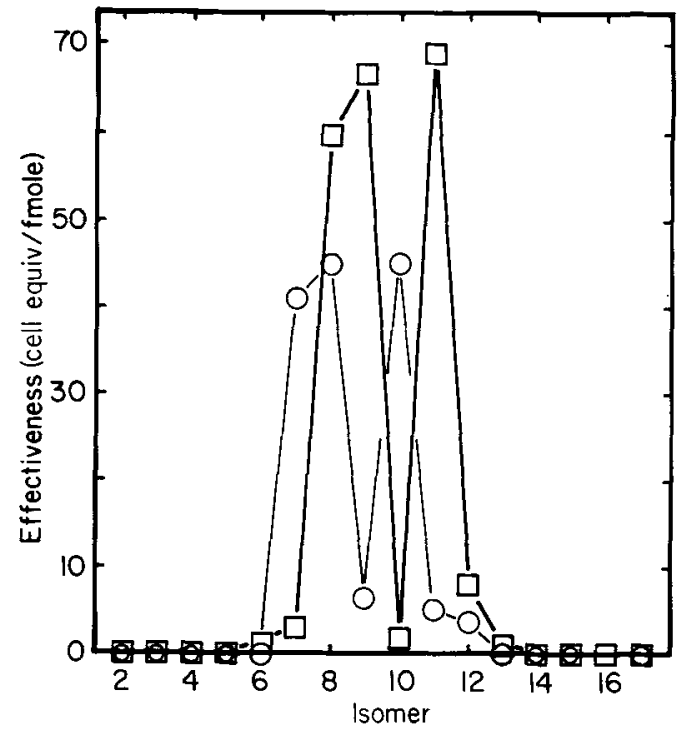

FIG. 7. Comparison of cyclopropane acids with acetylenic acids. Efficiencies of the cis-methylenoctadecanoic acid isomers ( $\square-\square$ ) compared to those obtained previously (11) for the octadecynoic acid isomers $(0-0)$ in supporting growth of the $E$. coli mutants at $37 \mathrm{C}$ with $1 \%$ glucose as the energy source.

phenomenon when examining acyl-CoA: phospholipid acyltransferases (27). In that work, the 9-ynoic acid was metabolically equivalent to and could be oriented in a manner that is spatially equivalent to the 10 -isomer. of cis-octadecenoate. The enzyme(s) appeared to interact in a highly selective manner with a cis. rotamer (rotational conformer) of the individual, solvated acetylenic acyl-CoA derivatives. Space-filling models readily demonstrated that the cisrotamers of the 7,8 and 10 acetylene analogs are spatially equivalent to the corresponding 8,9 and 11 cyclopropane isomers to which they are also nutritionally equivalent (Fig. 7). The appearance of a highly selective isomeric difference in the present study leads us to suggest that the irregular pattern of nutrient effectiveness obtained with cells in the glucose medium probably reflects some specific configurational acyl chain-protein interactions. At the present time, all studies of fatty acid transport, activation, esterification and hydrolysis in $E$. coli have failed to exhibit a specificity that accounts for the highly selective interactions reported here.

Since many of the cis-ethylenic acids were more effective nutrients in glucose than their spatially-equivalent cyclopropane analogs, some feature of the cis-ethylenic bond appears to allow them to be more consistently effective. If the required feature was merely the presence of $\pi$-bonds, as described earlier for 1-acylGPC:acyl-CoA acyltransferase activity (28), we might expect the acetylenic isomers to serve equally well as the cis-ethylenic analogs. The fact that they do not indicates that both spatial and electronic aspects are important features. Prior studies by Silbert et al. (29) noted that some cyclopropane acids were suitable nutrients for auxotrophic cultures, although Cronan et al. later concluded (30) that fatty acids of this type appear to impede exponential growth. Speculations on the impact of the cyclopropane acids on cell physiology were reviewed recently (31) without a definite role being demonstrated. Our results clearly indicate that different positional isomers of these acids can have highly selective effects, and that the presence or absence of $\pi$-bonds may indeed influence a cell's response to an acyl chain.

The use of a series of positional isomers has provided a range of cellular responses to a given type of acyl chain substituent. Not all cyclopropane acids are defective growth promotors, and some acetylenic acids support growth even though they have high melting points. General conclusions regarding the effect that a type of acid (e.g., cyclopropane or trans) may have upon cell physiology are not reliable when based on observations of only one or two members of a series. Furthermore, although all fatty acids will influence the membrane fluidity, effects other than fluidity can occur.

\section{Acyl Chain Adequacy in Different Media}

Although the growth on glucose was low with the $5,6,7,10,12$ and 13 isomers, the high growth observed with added cAMP or with glycerol indicates that there was nothing about the chemistry of those acyl chains per se that prevented their phospholipid derivatives from supporting cell functions. In these later media, the cyclopropane acids had $\epsilon$ values comparable to their corresponding cis-ethylenic analogs, which correlated well with the relative contribution that each isomeric acid can make to phospholipid fluidity (12). The close agreement with these two types of structural analogs indicates that the expected similarity in physical properties of cis-ethylene and cis-methylene prevails in the membrane lipids even though a different nutrient effectiveness is observed. In addition, the mole \% values for the cyclopropane acids in the presence of CAMP are similar to those in the absence of cAMP. This result leads to similar estimated functionality factors in both media. Such a result suggests that the cyclic nucleotide enhanced cell growth without appreciably altering the acyl chain's proportional contribution to membrane fluidity. 
Regular "bell-shaped" responses to different positional isomers were observed more consistently with the eukaryote, $S$. cerevisiae, than with the prokaryote, $E$. coli. For this reason, we conclude that the plasticity or degree of expansion of the membrane lipids, which follows the same "bell-shaped" pattern, is a principal factor limiting yeast cell growth with these acids. This pattern of results was also obtained for cis-octadecenoate isomers with KD-115 grown with either glucose (repressed) or glycerol (depressed) as the carbon source (24). Thus, the eukaryotic yeast cells have fewer growth-limiting selective interactions with nutrient fatty acids than do the bacteria studied, and they seem more resistant than $E$. coli to this aspect of glucose repression. We have reported, however, that the yeast mutant KD-46 showed highly selective inhibitory effects of isomeric trans-octadecenoates, whereas the bacteria appeared unresponsibe to the positional differences among the trans fatty acids (13). Thus, highly selective interactions of acyl chains are also clearly evident in yeast.

\section{Modulation of Catabolite Repression}

The ability of some fatty acids to support greater bacterial growth with glycerol than with glucose is an indication that catabolite repression may be involved. Repression of cellular functions by glucose (32) may occur by reduction of the cellular content of CAMP (33) although an alternative accumulation of a repressive factor has been reported (34). The wellrecognized action of CAMP in combination with catabolite activator protein (35) in stimulating transcription of some genes is paralleled by an ability of unadenylyated glutamine synthetase to stimulate transcription in the case of the enzymes of the histidine utilizing (hut) system (36). In this case, both glucose and ammonia can repress the formation of the hut enzymes, and reduction of the ammonium concentration allows release of the system from the glucose (catabolite) represssion (37). A related example is the synthesis of tyramine oxidase which is also relieved from catabolite repression under conditions of nitrogen limitation (38) perhaps involving a factor other than glutamine synthetase. Our present results suggest that general metabolic regulation by carbohydrates (energy sources) and ammonia and amino acids (nitrogen sources) may be complemented by effects of lipids. Fatty acids or their intracellular derivatives appear to modulate or mediate a form of catabolite repression in which cell growth is not possible with certain types of fatty acids and is fully competent with others.

The failure of cells to grow indicates that some fatty acids can selectively create a serious inbalance in the regulatory systems of the cell. A pleiotropic effect on transport systems of $E$. coli $\mathrm{K}-1061$ was described (39) for cells grown in glucose with high levels of cis-11-octadecenoate or cis-9-methylenehexadecanoate. The manner in which the fatty acids were involved in the response of catabolite repression was not clear, and oleate (c9-18:1), palmitoleate (c9-16:1) and cis-7-hexadecenoate gave normal transport activity (39). In those experiments, no reduction in the growth of the cells was observed in contrast to the present report. An earlier report (19) noted that an $E$. coli auxotroph could grow in glycerol on 9-cis, 9-trans, 9-acetylenic and 11-cyclopropane acids, but only on 9-cis and 11-cyclo in glucose. Subsequent experiments focused attention on the physical properties of the different chemical types of acid and have used mutant variants that may not have the regulatory features of the parent mutant. Our results suggest that a wide variety of variants permitting growth on certain fatty acids could occur by spontaneous mutations in the pleiotropic cAMP regulatory system.

Our current study documents a highly selective effect and indicates the conformation of isomer and chemical type of acyl chain capable of creating the impaired growth condition. Further work is needed to identify the enzyme activities that are directly affected by this specific regulatory response to fatty acids or their intracellular derivatives. The current findings are of particular importance in regard to how different acyl chains may influence cell physiology since they show that cellular cyclic nucleotides can alter, and may mediate, markedly different effects produced by closely related fatty acids. These different regulatory effects of fatty acids can be expected to occur in other cells to varied degrees and to be more pronounced under conditions of catabolite repression.

\section{REFERENCES}

1. Chapman, D., Q. Rev. Biophys. 8:185 (1975).

2. Keith, A.D., B.J. Wisnieski, S. Henry, J.C. Williams, in "Lipids and Biomembranes of Eukaryotic Microorganisms," Edited by J.A. Erwin, Academic Press, New York, 1973, pp. 259-321.

3. Silbert, D.F., Ann. Rev. Biochem. 44:315 (1975).

4. Gurr, M.I., in "Biochemistry of Lipids," Edited by T.W. Goodwin, Buttersworths, London, 1974, pp. 181.

5. Lands, W.E.M., and C.G. Crawford, in "The Enzymes of Biological Membranes," Edited by A. Mortonosi, Plenum Press, New York, 1976, pp, $1-86$.

6. Sammuelsson, B., E. Granström, K. Green, M. Hamberg, and S. Hammarström, Ann. Rev. Bio- 
chem. $44: 669$ (1975).

7. Holman, R.T., in "Progress in the Chemistry of Fats and Other Lipids, Edited by R.T. Holman, Vol. 9, Pergamon Press, London, 1970, p, 607.

8. Barber, E., and W.E.M. Lands, J. Bacteriol. $115: 543$ (1973).

9. Wisnieski, B.J., and R.K. Kiyomoto, J. Bacteriol. $109: 186(1972)$.

10. Fox, C.F., Proc. Natl. Acad. Sci. USA 63:850 (1969).

11. Lands, W.E.M., J.B. Ohłrogge, J.R. Robinson, R.W. Sacks, J.A. Barve, and F.D. Gunstone, Biochìm. Biophys. Acta 485:451 (1977).

12. Ohlrogge, J.B., E.D. Barber, W.E.M. Lands, F.D. Gunstone, and I.A. Ismail, Can. J. Biochem. $54: 736$ (1976).

13. Vandenhoff, G., F.D. Gurstone, J. Barve, and W.E.M. Lands, J. Biol. Chem. 250:8720 (1975)

14. Linden, C.D., K.L. Wright, H.M. McConnell, and C.F. Fox, Proc. Natl. Acad. Sci. USA 70:2271 (1973).

15. Wilson, G., S.P. Rose, and C.F. Fox, Biochem. Biophys. Res. Commun. 38:617 (1970).

16. Cronan, J.E., Jr., C.H. Birge, and Vagelos, P.R., J. Bacteriol, 100:601 (1969).

17. Epstein, W., and C.F. Fox, J. Bacteriol. 103:274 (1970).

18. Wilson, G., and C.F. Fox, J. MOl. Biol, 55:49 (1971).

19. Schairer, H.U., and P. Overath, J. Mol. Biol. 44:209 (1969).

20. Christie, W.W., F.D. Gunstone, I.A. Ismail, and L. Wade, Chem. Phys. Lipids 2:196 (1968).

21. Resnick, M.A., and R.K. Mortimer, J. Bacteriol. 92:597 (1966).

22. Bard, M., R.A. Woods, and J.M. Haslam, Biochem. Biophys. Res. Commun. 56:324 (1974)

23. Keith, A.D., M. Resnick, and A.B. Haley, J. Bac- teriol. $98: 415$ (1969).

24. Walenga, R.W., and W.E.M. Lands, J. Biol. Chem. 250:9121 (1975).

25. Holub, B.J., and W.E.M. Lands, Can, J. Biochem. 53:1262 (1975).

26. Barton, P.G., and F.D. Gunstone, J. Biol. Chem. $250: 4470(1975)$

27. Tamai, Y., W.E.M. Lands, J.A. Barve, and F.D. Gunstone, Biochim. Biophys. Acta 296:563 (1973).

28. Okuyama H., and W.E.M. Lands, J. Biol. Chem. 244:6514 (1969).

29. Silbert, D.F., F. Ruch, and P.R, Vagelos, J. Bacteriol. $95: 1658$ (1968).

30. Cronan, J.E., Jr., W.D. Nunn, and J.G. Batchelor, Biochim. Biophys. Acta 348:63 (1974).

31. Taylor, F., and J.E. Cronan, Jr., J, Bacteriol. $125: 518(1976)$

32. Magasanik, B., Cold Springs Harbor Symp. Quant. Biol. 26:249 (1961)

33. Makman, R.S., and E.W. Sutherland, J. Biol Chem. 240:1309 (1976).

34. Ullman, A., F. Tillier, and J. Monod, Proc. Natl. Acad. Sci. USA 73:3476 (1976).

35. Zubay, G., D. Schwartz, and J. Beckwith, Proc. Natl. Acad. Sci USA 66:104 (1970).

36. Streicher, S.L., R.A. Bender, and B. Magasanik, J. Bacteriol. 121:320 (1975).

37. Prival, M.J., and B. Magasanik, J. Biol. Chem. 246:6288 (1971)

38. Okamura, H., Y. Murooka, and T. Harada, J. Bacteriol. 127:24 (1976).

39. Cox, G.S., H. Weissbach, and H.R. Kaback, J. Biol. Chem. 250:4542 (1975).

[Received June 14, 1978] 